\title{
Modeling the emetic potencies of food-borne trichothecenes by benchmark dose \\ methodology
}

\section{Denis Male ${ }^{\mathrm{a}^{*}}$, Wenda $\mathrm{Wu}^{\mathrm{a}, 1^{*}}$, Nicole J. Mitchell ${ }^{\mathrm{a}}$, Steven Bursian ${ }^{\mathrm{b}}$, James J. Pestka ${ }^{\mathrm{a}}$, and Felicia $\mathbf{W u}^{\text {å }}$}

${ }^{\mathrm{a}}$ Department of Food Science and Human Nutrition, Michigan State University, 469 Wilson Rd, East Lansing, MI, 48824, USA; ${ }^{b}$ Department of Animal Science, Michigan State University, 474 S. Shaw Lane, East Lansing, MI, 48824, USA

*Co-first authors

${ }^{£}$ Corresponding author email: fwu@msu.edu; phone: 517-352-4442

\begin{abstract}
Trichothecene mycotoxins commonly co-contaminate cereal products. They cause immunosuppression, anorexia, and emesis in multiple species. Dietary exposure to such toxins often occurs in mixtures. Hence, if it were possible to determine their relative toxicities and assign toxic equivalency factors (TEFs) to each trichothecene, risk management and regulation of these mycotoxins could become more comprehensive and simple. We used a mink emesis model to compare the toxicities of deoxynivalenol, 3-acetyldeoxynivalenol, 15acetyldeoxynivalenol, nivalenol, fusarenon-X, HT-2 toxin, and T-2 toxin. These toxins were

\footnotetext{
1‘Permanent Address' College of Veterinary Medicine, Nanjing Agricultural University, No. 1 Weigang, Nanjing, 210095, People's Republic of China
} 
administered to mink via gavage and intraperitoneal injection. The United States Environmental Protection Agency (EPA) benchmark dose software was used to determine benchmark doses for each trichothecene. The relative potencies of each of these toxins were calculated as the ratios of their benchmark doses to that of DON. Our results showed that mink were more sensitive to orally administered toxins than to toxins administered by IP. T-2 and HT-2 toxins caused the greatest emetic responses, followed by FX, and then by DON, its acetylated derivatives, and NIV. Although these results provide key information on comparative toxicities, there is still a need for more animal based studies focusing on various endpoints and combined effects of trichothecenes before TEFs can be established.

Keywords: mycotoxins, deoxynivalenol, emesis, toxic equivalency factor 


\section{INTRODUCTION}

Trichothecene mycotoxins, produced most commonly by the fungi Fusarium graminearum, $F$. culmorum, and $F$. sporotrichioides, often contaminate common cereal grains such as wheat, barley, rye, and oats (Foroud and Eudes, 2009; Stanciu et al., 2015). The most commonly occurring and regulated trichothecene is deoxynivalenol (DON), also called "vomitoxin," as it induces emesis in multiple species. The United States Food and Drug Administration (FDA) has set an industry guideline for the maximum allowable concentration of DON at $1 \mathrm{mg} / \mathrm{kg}$ (FDA, 2010). Other regulatory bodies such as the European Commission have set similar or more stringent DON standards in food (European Commission, 2006).

DON is one of the trichothecenes that make up the sub-class type B trichothecenes. The type B trichothecenes are characterized by a keto group at carbon- 8 of the parent epoxy-trichothecene nucleus. This group includes five associated congeners: DON, its acetylated derivatives 15acetyldeoxynivalenol (15-ADON) and 3-acetyldeoxynivalenol (3-ADON), nivalenol (NIV) and its acetylated derivative fusarenon X (FX) (Fig. 1). In addition to the type B trichothecenes, there are those trichothecenes classified as type A, of which T-2 and HT-2 toxins are the most toxic. Type A trichothecenes are characterized by the presence of a hydroxyl group, ester function, or no oxygen substitution at carbon-8 (McCormick et al., 2011). DON and its related trichothecene mycotoxins cause a variety of adverse effects in multiple species. These adverse effects have been reviewed by Pestka et al. (2010a, 2010b) and include emesis, nausea, anorexia, diarrhea, growth retardation, neuroendocrine effects, and disruption of the immune system. The primary clinical signs associated with exposure to DON and related trichothecenes in human populations are nausea and vomiting. Therefore, these specific effects should be the endpoints utilized in risk assessment for human consumption of trichothecenes (Luo, 1994; Yoshizawa, 1983). 
Gastroenteritis outbreaks in the U.S. and abroad have been associated, inconclusively, with DON contamination of foods. From October 1997 to October 1998 there were 16 outbreaks of gastrointestinal illness affecting more than 1,900 school children following the ingestion of burritos from two unrelated companies (Steinberg et al., 2006). The children who consumed the burritos suffered nausea, headache, abdominal cramps, vomiting, and diarrhea. Laboratory analysis did not find contamination with common bacterial strains associated with gastroenteritis, suggesting that the symptoms observed were due to a toxin contamination. Some burrito samples had detectable levels of DON, although they were below the FDA regulatory guideline of 1 mg/kg. Outbreaks in China from 1984 to 1991 were linked to moldy cereal grains. DON, as well as other trichothecenes, were verified in samples taken during this time period, and found at concentrations ranging from 2 to $50 \mathrm{mg} / \mathrm{kg}$ (Pestka and Smolinski, 2005). Several thousand individuals from the Kashmir Valley of India were affected with gastroenteritis from consumption of foods made with moldy wheat. Samples were found to contain DON at 0.34 to $8.4 \mathrm{mg} / \mathrm{kg}$ (Bhat et al., 1989). It is important to note, that although food samples may contain trichothecene levels below the FDA guideline, the threshold for human emesis is still unknown and that this threshold might also vary greatly due to differences in age, sex, diet, health status, genetic differences, etc. making it difficult to assess this value (Stadler et al., 2003).

The Joint Expert Committee on Food Additives of the Food and Agriculture Organization and World Health Organization (JECFA) has determined a recommended acute reference dose (ARfD) for DON and its acetylated derivatives of $8 \mu \mathrm{g} / \mathrm{kg}$ bw/day. Although JECFA has not set a similar ARfD for NIV and FX, the European Food Safety Authority (EFSA) has made a recommendation for a joint tolerable daily intake of $1.2 \mu \mathrm{g} / \mathrm{kg}$ bw/day (EFSA, 2013). JECFA has 
established a provisional maximum tolerable daily intake (PMTDI) of $60 \mathrm{ng} / \mathrm{kg} \mathrm{bw} / \mathrm{day}$ for either T-2 toxin alone or a mixture of T-2 and HT-2 (JECFA, 2001).

Although trichothecenes co-occur in numerous food commodities, a lack of robust toxicology and epidemiology studies makes it difficult to determine appropriate regulatory levels for each individual trichothecene. Additionally, risk assessment of foods with potential co-contamination is extremely difficult when there are limited studies on the toxicity of mixtures. Since there is a lack of consensus among governmental agencies on regulation of trichothecenes, assigning each trichothecene a toxic equivalency factor (TEF) would be desirable for risk assessment and regulatory purposes. Using such an approach, a single regulatory standard could be set for the sum total of all co-occurring trichothecenes in cereals and their products.

TEF values were first introduced in the practice of regulatory risk assessment for the polychlorinated dibenzodioxins (PCDDs), the polychlorinated biphenyls (PCBs), and the polychlorinated dibenzofurans (PCDFs) (Van den Berg et al., 1998; Van den Berg et al., 2006). The theory behind associated TEF values is that, because compounds in a particular group (e.g., dioxins) usually co-occur, are structurally similar, have similar modes of toxicity, and have additive impacts, they are each assigned an equivalency factor in comparison to the toxicity value of a reference compound. Although there is limited evidence for the additive effect of trichothecenes when consumed as mixtures, the initiation of the process to develop a similar regulatory mechanism to that of the TEF values of PCDDs, PCBs, and PCDF is warranted and essential to future risk assessment practices for these similar, co-occurring food mycotoxins.

Previously, the relative anorectic potencies of the above-mentioned trichothecenes were calculated by Male et al. (2015), using raw data collected from a mouse anorexia study (Wu et 
al., 2012a,b). In that analysis, two methods were used to rank the potency of each trichothecene relative to DON based on feed refusal: 1) benchmark dose (BMD) analysis and 2) incremental area under the curve (IAUC). The mouse model cannot be used to study emetic responses to trichothecenes, because mice are incapable of vomiting. Therefore, the present study applied the same principles, with a new set of data from a mink model to utilize emesis as the outcome variable. Currently, the BMD is used by regulatory bodies worldwide as the "point of departure" from which to calculate tolerable daily intakes or reference doses for humans. The BMD methodology allows for use of animal data with limited number of dose groups and small $n$ values. Here, DON, 3-ADON, 15-ADON, NIV, FX, HT-2, and T-2 toxins were ranked by potency to induce emesis, based on their individual BMD values following oral gavage and intraperitoneal (IP) dosing in mink. Relative potencies were assigned to each toxin in relation to DON. This work is an important initial step in developing a uniform risk assessment strategy for complex mixtures of trichothecenes.

\section{MATERIALS AND METHODS}

\subsection{Mink feeding and emesis trials}

The raw emesis data used in this analysis were collected from prior mink experiments. The experimental design and procedures, used in the emesis trials (Fig. 2), were previously described by Wu et al. (2012a). Briefly, DON, 3-ADON, 15-ADON, NIV, FX, HT-2, and T-2 toxins with purity of $>98 \%$ were either given by gavage or IP dosing. A total of 60 standard dark, female mink between 12 and 24 months of age were obtained from the Michigan State University Experimental Fur Farm. The animals were bred and housed in accordance with the 2010 Fur 
Commission guidelines. Animal treatment was in accordance with the NIH guidelines and the research was approved by the Michigan State University Institutional Animal Care and Use Committee (MSU-IACUC). All animals were acclimated for 1 week, and then fasted for $24 \mathrm{~h}$ before initiating the experiment. To maintain a constant volume of gastric constituents in the experimental animals, water was provided ad libitum.

For IP dosing studies (Fig. 2), mink were given $50 \mathrm{~g}$ of feed after $24 \mathrm{~h}$ of fasting. Thirty minutes after feeding, animals were given either phosphate buffered saline or doses of toxins in a volume equivalent to $1 \mathrm{ml} / \mathrm{kg}$ bw/day via IP injection. The animals were then monitored for emesis for 6 h. The latency to emesis, incidence of emesis, duration of emesis, and number of emetic events including retching and vomiting were recorded (Table 1). For gavage dosing studies (Fig. 2), 50 $\mathrm{g}$ of feed was provided to mink after $24 \mathrm{~h}$ of fasting. Thirty minutes following feeding, mink were given either saline or toxin in a volume equivalent to of $1 \mathrm{ml} / \mathrm{kg}$ bw/day via gavage. The animals were then monitored for emesis for $3 \mathrm{~h}$ and the data were recorded (Table 2).

\subsection{Benchmark dose modeling and calculation of relative emetic potency}

The United States Environmental Protection Agency (EPA) (Larypoor et al., 2013) benchmark dose software version 2.6.0.1 (BMDS 2.6.0.1) was used to model and calculate the BMD of each mycotoxin. The raw data, including the doses, number of animals per dose group, and number of animals that vomited were entered into an in-built excel wizard (Version 1.10) for dichotomous data. The variable from Wu et al. (2012a) utilized to calculate the BMD values were "incidence" for IP (Table 1) and gavage (Table 2) at the various doses. The BMD was determined as the point of departure at a benchmark response of $10 \%\left(\mathrm{BMR}_{10}\right)$. We calculated the averaged $\mathrm{BMD}_{10}$ 
values and $95 \%$ confidence interval for the incidence of emesis for each trichothecene from results of parametric bootstrapping, using 500 iterations. The $\mathrm{BMDL}_{10}$ was derived from the lower one-sided 95\% confidence interval of the distribution for each dose group (Crump, 1995; Deutsch, 2012).

Dose-response data for each toxin were fit to several built-in mathematical models in BMDS, including gamma, dichotomous-Hill, logistic, log-logistic, probit, log-probit, Weibull, multistage $2^{\circ}, 3^{\circ}$, and $4^{\circ}$ cancer, and quantal linear models. The model that provided the best fit for the experimental dose-response data was selected based on the ratio of BMD to BMDL (a ratio closer to 1 indicates lower uncertainty and variability), Akaike Information Criterion (AIC), goodness-of-fit $p$ values, scaled residuals for dose groups near the BMD calculated, visual inspection of graphical outputs (fit of curve to dose-response points) and whether there were any BMDS model warnings (EPA, 2012). The BMD analysis procedure was done for all the trichothecenes studied, and their relative emetic potencies were calculated as the ratio of the BMD of DON to the BMD of each of the other trichothecenes. The procedure for calculating relative potency was described in our previous publication (Male et al., 2015).

\subsection{Fixed dose comparison of emetic events}

Additionally, we summarized and compared the number of emetic events ("total" under "number of emetic events" in Table 2) following oral administration of $0.5 \mathrm{mg} / \mathrm{kg}$ bw of each of the toxins. This dose was selected since it was the common dose in all investigations. These values, "total number of emetic events", for all the trichothecenes were divided by that of DON to determine their relative potencies. 


\section{RESULTS}

\subsection{IP dosing experiments}

The IP dosing BMDs for the type B trichothecenes were 73, 108, 141, 63, and $60 \mu \mathrm{g} / \mathrm{kg}$ bw for DON, 15-ADON, 3-ADON, NIV, and FX, respectively. The BMDs for HT-2 and T-2 toxins administered by IP injection were both equal to $31 \mu \mathrm{g} / \mathrm{kg}$ bw and identical (Table 3), suggesting that they were equally emetic to mink through this exposure route. The BMD, or minimum amount of toxin required to induce vomiting in mink by IP injection, was greatest for 3-ADON (i.e., 3-ADON was the least emetic); followed in decreasing order by 15-ADON, DON, NIV, FX, and HT-2 or T-2 toxins. The relative potencies of the trichothecenes were calculated as the ratio of the BMD of DON to that of each of the other toxins. Thus, the calculated rank order of the IP exposure emetic potencies was: HT-2/T-2, followed in decreasing order by FX, NIV, DON, 15-ADON, and 3-ADON (Table 3). Moreover, the emetic potency of DON was about 1.7 times higher than that of either 3-ADON or 15-ADON. Generally, the BMDs of the group of DON, 3-ADON and 15-ADON were higher than the BMDs of NIV and its acetylated derivative FX, implying that DON and its derivatives had a lower relative emetic potency than either NIV or FX. The order of potency based on IP administration is: HT- $2 \approx \mathrm{T}-2>\mathrm{FX}>\mathrm{NIV}>\mathrm{DON}>$ $15-\mathrm{ADON}>3-\mathrm{ADON}$.

\subsection{Gavage dosing experiments}

The oral (gavage) BMDs for the type B trichothecenes were 24, 40, 198, 141, and $23 \mu \mathrm{g} / \mathrm{kg}$ bw for DON, 15-ADON, 3-ADON, NIV, and FX, respectively. The rank order of the calculated emetic potency of each toxin was FX, DON, 15-ADON, NIV, and 3-ADON, respectively; with FX being the most toxic type B trichothecene and 3-ADON the least toxic (Table 3). The oral 
exposure emetic BMD of both T-2 and HT-2 toxins was $14 \mu \mathrm{g} / \mathrm{kg}$ bw; it was lower than the BMDs of all type B trichothecenes, indicating a greater toxicity of T-2 and HT-2 toxins. Via oral exposure, DON was 8.3 and 1.7 times more potent than 3-ADON and 15-ADON, respectively. Unlike IP dosing where the BMD of 3-ADON was slightly higher than that for 15-ADON (141 and $108 \mu \mathrm{g} / \mathrm{kg}$ bw respectively), for oral administration, the BMD for 3-ADON was 5 times the value for 15-ADON (198 and $40 \mu \mathrm{g} / \mathrm{kg}$ bw respectively). The oral emetic potencies of NIV and FX relative to DON were 0.17 and 1.04 respectively, indicating that the potential for FX to induce emesis in mink was 6 times that of NIV. The order of potency based on gavage is: HT-2 $\approx$ $\mathrm{T}-2>\mathrm{FX}>\mathrm{DON}>15-\mathrm{ADON}>\mathrm{NIV}>3-\mathrm{ADON}$.

\subsection{Comparing emetic potency for IP and gavage routes of exposure}

The BMD analysis also revealed interesting findings about the two routes of exposure. Generally, the emetic potency of the trichothecenes was higher via the gavage route than via IP injection. With the exception of NIV and 3-ADON whose IP injection BMD values were lower, the calculated values for IP exposure were higher for T-2, HT-2, FX, DON, and 15-ADON than those calculated when the trichothecenes were administered by gavage. For HT-2 and T-2 toxins, the BMDs were 31 and $14 \mu \mathrm{g} / \mathrm{kg}$ bw for IP and gavage dosing, respectively. 3-ADON had the largest BMD values for either exposure routes, thus the lowest potency of the common dietary trichothecenes. The findings further show that irrespective of exposure route, DON is more toxic than both 15-ADON and 3-ADON. The oral emetic potency of FX is 6 times higher than that of NIV. However, there was no difference in toxicity between the two toxins when introduced to

mink by IP injection, because their emetic potencies were the same. The potency of NIV relative to DON is 1.22 when given by IP dosing; but when administered orally, DON is 6 times more potent than NIV. 


\subsection{Comparing the emetic events from oral exposure to a fixed dose of trichothecenes}

The number of emetic responses to a fixed oral dose $-0.5 \mathrm{mg} / \mathrm{kg}$ bw- of type A and type B trichothecenes is summarized in Table 4 (Wu et al., 2012a). Type A trichothecenes caused the most incidents of emesis as compared to their type B counterparts. The sum of the frequencies of retching and vomiting were not significantly different for HT-2 and T-2 toxins. Among the type B trichothecenes, DON produced the highest number of responses followed in decreasing order by NIV, FX, 15-ADON, and 3-ADON. The number of incidents caused by FX, DON and NIV were not significantly different. Similarly, the sum of emetic events caused by $15-$ ADON and 3ADON did not differ significantly. The total number of emetic events produced by FX, DON, or NIV was significantly higher than the totals for either 15-ADON or 3-ADON (Table 4). In summary, the rank order of total emetic events produced by each toxin when a uniform dose equal to $0.5 \mathrm{mg} / \mathrm{kg}$ bw was administered was $\mathrm{HT}-2 \approx \mathrm{T}-2>\mathrm{DON} \approx \mathrm{FX} \approx \mathrm{NIV}>15-\mathrm{ADON} \approx 3$ ADON.

\section{DISCUSSION}

As more mycotoxins are being regulated in foods worldwide, challenges arise when setting appropriate standards for each. Because the trichothecene mycotoxins are produced by the same groups of fungi, co-contaminate the same cereal crops, and cause largely the same adverse health effects, regulation of these mycotoxins can be simplified by setting TEFs relative to a single toxin. This is the first study to compare the emetic potencies of trichothecenes, using the BMD method, to find appropriate points of departure for risk assessment purposes and to develop relative potencies for each toxin relative to DON. 
In the case of emesis from trichothecene exposure, we found that the animals were more sensitive to gavage than to IP administration. This is because the vomiting center in the brain is stimulated when either the receptors in the periphery (gastrointestinal tract) or in the blood are activated by the presence of toxins (Horn, 2008; Prelusky and Trenholm, 1993). Studies have shown that trichothecenes cause gastroenteritis (Pestka, 2010), which activates the enterochromaffin cells in the epithelium to release 5-hydroxytryptamine (5-HT) or serotonin. The 5-HT neurotransmitter sends signals to the brain vomiting center to induce vomiting (Prelusky and Trenholm, 1993). Likewise, introduction of toxins into the blood by IP dosing or absorption from the gut into the systemic circulation can stimulate the vomiting center via direct activation of $5-\mathrm{HT}_{3}$ receptors in the chemoreceptor trigger zone (CTZ) in the brain (Becker, 2010; Dietrich et al., 2015; Kovac, 2016; Horn, 2008; Lang, 1999; Prelusky and Trenholm, 1993). This suggests that oral exposure could result in double stimulation of the vomiting center i.e. via 5-HT receptors in the gut before absorption, and via 5- $\mathrm{HT}_{3}$ receptors in the $\mathrm{CTZ}$ during systemic circulation, eliciting a higher emetic response than via IP exposure. The IP route induces vomiting via only the CTZ, which may explain the lower emetic potency as compared to the oral route. Human consumption of trichothecene contaminated foods is an oral exposure making the relative potencies of the gavage model more appropriate for human risk assessment.

The oral potency decreased from $\mathrm{DON}$ to $15-\mathrm{ADON}$ to $3-\mathrm{ADON}$, respectively. This result is consistent with other studies that demonstrated that acetylation of DON at carbon-3 (C-3) to 3ADON decreases toxicity (Alexander et al., 1999; Kimura et al., 1998; McCormick, 2013; Zhou et al., 2008). In contrast to a number of previous studies that indicated that acetylation of DON at C-15 to 15-ADON increases cytotoxicity (Desjardins et al., 2007), this study showed that the emetic potency of DON was greater than 15-ADON. The low toxicity of 15-ADON in vivo 
could be partly due to deacetylation to DON in the intestine prior to absorption (Veršilovskis et al., 2012) and in part, attributed to faster rates of clearance as compared to DON (Broekaert et al., 2015). In pigs, the rapid and nearly complete pre-systemic hydrolysis (99\%) of 15-ADON to DON was observed. However, the rates of clearance were in increasing order: 3 -ADON > 15ADON > DON (Broekaert et al., 2015). Although both 3-ADON and 15-ADON ultimately undergo hydrolysis in the intestine before absorption, each toxin (DON, 3-ADON, and 15ADON) exerts a different toxicity on the local tissue (Broekaert et al., 2015). For example, Pinton et al. (2012) used in vitro, ex vivo and in vivo studies to compare the effects of DON, 3ADON and 15-ADON on the barrier function of intestinal cells and activation of MAPK. The study revealed that $15-\mathrm{ADON}$ caused more severe effects including histological lesions, activation of MAPK and decreased expression of tight junction proteins than DON and 3-ADON (Pinton et al., 2012).

IP dosing results show that the BMD for NIV and FX are similar i.e. 60 and $63 \mu \mathrm{g} / \mathrm{kg}$ bw respectively. The similar emetic potency could be explained by reports that FX is metabolized to NIV in the liver and kidney after absorption, suggesting that NIV mediates FX's in vivo toxicity (Poapolathep et al., 2003). Our results further indicated that the oral exposure potency of FX was equivalent to that of DON, but 6 times higher than that of NIV. This observation is contrary to previous in vitro studies that have reported higher toxicities for NIV and FX as compared to DON (Abbas et al., 2013; Eriksen et al., 2004; Q. Wu et al., 2013). The higher rate of absorption for DON (Avantaggiato et al., 2004; Kongkapan et al., 2016; Pralatnet et al., 2015) could counterbalance the differences in emetic potency. The higher toxicity of FX as compared to NIV is consistent with an earlier finding that FX was more efficiently absorbed than NIV (Poapolathep et al., 2003). The limited absorption of NIV following oral gavage could have 
reduced its emetic potency, because a large amount of NIV might have passed through the gastrointestinal tract without being absorbed (Poapolathep et al., 2003). Studies have shown that the presence of certain functional groups at C-4 of type B trichothecenes also influences their toxicity. For instance, at the C-4 position, the toxicity of the groups is in the order: acetyl > hydroxyl > hydrogen groups (Zhou et al., 2008). Additionally, studies using A. thaliana leaf model showed that acetylation at the C-4 position increased cytotoxicity of trichothecenes (Desjardins et al., 2007). This would indicate that the change in the acetyl group of FX to a hydroxyl group in NIV would decrease FX's toxicity in vivo, making it more toxicologically similar to DON.

Type A trichothecenes T-2 and HT-2 had the highest emetic potencies among all the toxins. Although some studies have demonstrated that the toxicity of T-2 is greater than that of HT-2 toxin (Königs et al., 2009; Visconti et al., 1991), their emetic potencies were the same in the mink model. One possible reason is that T-2 toxin is rapidly bio-transformed to HT-2 toxin following oral exposure (Sintov et al., 1986; Q. Wu et al., 2013; Zhou et al., 2008). It is then rapidly absorbed as a mixture of T-2 and HT-2, suggesting that the observed emetic events in mink are a consequence of the absorbed HT-2 (JECFA, 2001; Conrady-Lorck et al., 1988; MuroCach et al., 2004).

The analysis of relative potencies conducted in this work and our previous publication (Male et al., 2015) are important aspects to developing a methodology to assess risk of food commodities with mixtures of these trichothecenes. It is important to analyze the differences in toxicity of each trichothecene in relation to exposure route and toxic endpoint. Here we have provided potency evaluations for emesis through two different types of data, BMD analysis of emetic incidence and comparison of total number of emetic events. In general, the ranking of potency 
based on these two separate data sets were similar. However, it is interesting to note that although the mink requires a significantly higher dose of NIV for initiation of an emetic response compared with DON and FX, they all induce a similar number of emetic events. Additionally, a significantly lower dose of 15-ADON compared with NIV and 3-ADON will induce emesis; however 15-ADON and 3-ADON given at a common dose will induce the same number of emetic events, which is less than the number of events induced by NIV. Male et al. (2015) observed a relative potency ranking of the trichothecenes of $\mathrm{T}-2>>\mathrm{FX}>\mathrm{HT}-2>\mathrm{NIV}>\mathrm{DON} \approx$ 3 -ADON $\approx 15$-ADON based on anorexia as an endpoint. This endpoint demonstrates that the potency of DON on emesis is significantly higher than its potency in regards to anorexia. When emesis is the observed endpoint DON acts more like FX than 15-ADON and 3-ADON. These factors need to be considered and the appropriate endpoint determined for future work.

We propose the use of the TEF approach in assessing the human health risk due to trichothecenes exposure in food. Although information about the mechanisms of action of some trichothecenes is incomplete since most available studies have focused on DON (Escrivá et al., 2015; Sobrova et al., 2010), the molecular structures of trichothecenes are similar enough to suggest similar mechanisms. Our data on emetic effects of each trichothecene in a mink model allow us to compare their toxicities through calculation of emetic potencies relative to DON. Another limitation to the TEF approach for trichothecenes is that very few studies have evaluated their potential interactions and consequent effects in animals and yet we know that these toxins typically co-occur in food (Bertuzzi et al., 2014; Desjardins et al., 2007; Ibáñez-Vea et al., 2012; Pronk et al., 2002; Šegvić Klarić, 2012). Although a recent investigation reported some synergistic toxic effects in vitro (Alassane-Kpembi et al., 2014), these are inconclusive since there is still uncertainty over the nature of interactions in vivo. Therefore, more research on 
emetic responses induced by mixtures of these mycotoxins using animal models is needed to inform risk assessment.

Taken together, the results of this study demonstrate that assigning of TEFs for trichothecenes that co-occur in food is possible. As mentioned earlier, for the method to be valid, the emetic effects of the compounds need to be additive (Van den Berg et al., 1998). Therefore, proper understanding of the relative potencies of mycotoxins that occur in mixtures is important for estimation of their overall toxicity.

\section{Acknowledgment}

This work was funded by USDA NIFA 2011-0635, USDA Wheat and Barley SCAB Initiative Award 59-0206-9-058, Public Health Service Grant ES03553 from the National Institutes of Health, the National Natural Science Foundation of China 31402268, the Fundamental Research Funds for the Central Universities KJQN201526, Natural Science Foundation of Jiangsu Province of China BK20140691, National Natural Science Foundation of China 31572576, the Priority Academic Development Program of Jiangsu Higher Education Institutions, the Borlaug Higher Education Agricultural Research and Development BHEARD/USAID CGA BFS-G-1100002, and the National Institute of Environmental Health Sciences of the Nation Institutes of Health T32ES007255. 


\section{REFERENCES}

Abbas, H.K., Yoshizawa, T., Shier, W.T., 2013. Cytotoxicity and phytotoxicity of trichothecene mycotoxins produced by Fusarium. Toxicon 74, 68-75.

Alassane-Kpembi, I., Puel, O., Oswald, I., 2014. Toxicological interactions between the mycotoxins deoxynivalenol, nivalenol and their acetylated derivatives in intestinal epithelial cells. Archives of Toxicology 89, 1337-1346.

Alexander, N.J., McCormick, S.P., Ziegenhorn, S.L., 1999. Phytotoxicity of selected trichothecenes using Chlamydomonas reinhardtii as a model system. Natural Toxins 7, 265-269.

Avantaggiato, G., Havenaar, R., Visconti, A., 2004. Evaluation of the intestinal absorption of deoxynivalenol and nivalenol by an in vitro gastrointestinal model, and the binding efficacy of activated carbon and other adsorbent materials. Food and chemical toxicology 42, 817-824.

Becker, D.E., 2010. Nausea, vomiting, and hiccups: a review of mechanisms and treatment. Anesthesia progress $57,150-157$.

Bertuzzi, T., Leggieri, M.C., Battilani, P., Pietri, A., 2014. Co-occurrence of type A and B trichothecenes and zearalenone in wheat grown in northern Italy over the years 2009-2011. Food Additives \& Contaminants: Part B Surveillance 7, 273-281.

Bhat, R., Ramakrishna, Y., Beedu, S., Munshi, K., 1989. Outbreak of trichothecene mycotoxicosis associated with consumption of mould-damaged wheat products in Kashmir Valley, India. The Lancet $333,35-37$.

Broekaert, N., Devreese, M., De Mil, T., Fraeyman, S., Antonissen, G., De Baere, S., De Backer, P., Vermeulen, A., Croubels, S., 2015. Oral Bioavailability, Hydrolysis, and Comparative Toxicokinetics of 3Acetyldeoxynivalenol and 15-Acetyldeoxynivalenol in Broiler Chickens and Pigs. Journal of Agricultural and Food Chemistry 63, 8734-8742. 
Conrady-Lorck, S., Gareis, M., Feng, X.-C., Amselgruber, W., Forth, W., Fichtl, B., 1988. Metabolism of T-2 toxin in vascularly autoperfused jejunal loops of rats. Toxicology and applied pharmacology 94, 23-33. Crump, K.S., 1995. Calculation of benchmark doses from continuous data. Risk Analysis 15, 79-89.

Desjardins, A.E., McCormick, S.P., Appell, M., 2007. Structure-activity relationships of trichothecene toxins in an Arabidopsis thaliana leaf assay. Journal of Agricultural and Food Chemistry 55, 6487-6492.

Deutsch, R.C., Piegorsch, W.W., 2012. Benchmark Dose Profiles for Joint-Action Quantal Data in Quantitative Risk Assessment. Biometrics 68, 1313-1322.

Dietrich, B., Ramchandran, K., Von Roenn, J.H., 2015. Nausea and Vomiting. Psycho-Oncology 3, 199208.

Eriksen, G.S., Pettersson, H., Lundh, T., 2004. Comparative cytotoxicity of deoxynivalenol, nivalenol, their acetylated derivatives and de-epoxy metabolites. Food and Chemical Toxicology 42, 619-624.

Escrivá, L., Font, G., Manyes, L., 2015. In vivo toxicity studies of fusarium mycotoxins in the last decade: A review. Food and Chemical Toxicology 78, 185-206.

European Commission, 2006. Commission Regulation (EC) No 1881/2006 of 19 December 2006. Setting maximum levels for certain contaminants in foodstuff. 2006R1881-EN-01.09. 2014-014.001-1.

http://eur-lex.europa.eu/legal-content/EN/ALL/?uri=CELEX\%3A32006R1881

Food and Drug Administration, United States (FDA), 2010. Guidance for industry and FDA: Advisory levels for deoxynivalenol (DON) in finished wheat products for human consumption and grains and grain by-products used for animal feed. US Food and Drug Administration: Silver Spring, MD, USA.

http://www.fda.gov/Food/GuidanceRegulation/GuidanceDocumentsRegulatoryInformation/ChemicalCo ntaminantsMetalsNaturalToxinsPesticides/ucm120184.htm

Foroud, N.A., Eudes, F., 2009. Trichothecenes in cereal grains. International Journal of Molecular Sciences 10, 147-173.

Horn, C.C., 2008. Why is the neurobiology of nausea and vomiting so important? Appetite 50, 430-434. 
Ibáñez-Vea, M., Lizarraga, E., González-Peñas, E., López de Cerain, A., 2012. Co-occurrence of type-A and type-B trichothecenes in barley from a northern region of Spain. Food Control 25, 81-88.

Joint Expert Committee on Food Additives and Contaminants (JECFA), 2001. T-2 and HT-2 toxins. Safety Evaluation of Certain Mycotoxins in Food. WHO Food Additives Series 47, 557-652. Kimura, M., Kaneko, I., Komiyama, M., Takatsuki, A., Koshino, H., Yoneyama, K., Yamaguchi, I., 1998. Trichothecene 3-O-Acetyltransferase Protects Both the Producing Organism and Transformed Yeast from Related Mycotoxins. Journal of Biological Chemistry 273, 1654-1661.

Kongkapan, J., Giorgi, M., Poapolathep, S., Isariyodom, S., Poapolathep, A., 2016. Toxicokinetics and tissue distribution of nivalenol in broiler chickens. Toxicon 111, 31-36.

Königs, M., Mulac, D., Schwerdt, G., Gekle, M., Humpf, H.-U., 2009. Metabolism and cytotoxic effects of T-2 toxin and its metabolites on human cells in primary culture. Toxicology $258,106-115$.

Kovac, A.L., 2016. Mechanisms of nausea and vomiting. Postoperative Nausea and Vomiting 1, 13-22.

Lang, I., 1999. Noxious stimulation of emesis. Digestive diseases and sciences 44, 58S-63S.

Larypoor, M., Bayat, M., Zuhair, M.H., Sepahy, A.A., Amanlou, M., 2013. Evaluation of The Number of CD4+ CD25+ FoxP3+ Treg Cells in Normal Mice Exposed to AFB1 and Treated with Aged Garlic Extract. Cell Journal (Yakhteh) 15, 37.

Luo, X., 1994. Food poisoning caused by Fusarium toxins, Proceedings of the Second Asian Conference on Food Safety. Chatuchak, Thailand: International Life Sciences Institute, p. 136.

Male, D., Mitchell, N., Wu, W., Bursian, S., Pestka, J., Wu, F., 2015. Modelling the anorectic potencies of food-borne trichothecenes by benchmark dose and incremental area under the curve methodology. World Mycotoxin Journal 9, 279-288.

McCormick, S.P., 2013. Microbial detoxification of mycotoxins. Journal of chemical ecology 39, 907-918. McCormick, S.P., Stanley, A.M., Stover, N.A., Alexander, N.J., 2011. Trichothecenes: from simple to complex mycotoxins. Toxins 3, 802-814. 
Muro-Cach, C.A., Stedeford, T., Banasik, M., Suchecki, T.T., Persad, A.S., 2004. Mycotoxins: Mechanisms of toxicity and methods of detection for identifying exposed individuals. Journal of Land Use \& Environmental Law 19, 537-556.

Pestka, J.J., 2010. Deoxynivalenol-induced proinflammatory gene expression: Mechanisms and pathological sequelae. Toxins 2, 1300-1317.

Pestka, J.J., Smolinski, A.T., 2005. Deoxynivalenol: toxicology and potential effects on humans. Journal of Toxicology and Environmental Health, Part B 8, 39-69.

Pinton, P., Tsybulskyy, D., Lucioli, J., Laffitte, J., Callu, P., Lyazhri, F., Grosjean, F., Bracarense, A.-P., Martine, K.-C., Oswald, I.P., 2012. Toxicity of deoxynivalenol and its acetylated derivatives on the intestine: differential effects on morphology, barrier function, tight junctions proteins and MAPKinases. Toxicological Sciences 130, 180-190.

Poapolathep, A., Sugita-Konishi, Y., Doi, K., Kumagai, S., 2003. The fates of trichothecene mycotoxins, nivalenol and fusarenon-X, in mice. Toxicon 41, 1047-1054.

Pralatnet, S., Poapolathep, S., Imsilp, K., Tanhan, P., Isariyodom, S., Kumagai, S., Poapolathep, A., 2015. The fate and tissue disposition of deoxynivalenol in broiler chickens. The Journal of Veterinary Medical Science 77, 1151.

Prelusky, D.B., Trenholm, H.L., 1993. The efficacy of various classes of anti-emetics in preventing deoxynivalenol-induced vomiting in swine. Natural toxins 1, 296-302.

Pronk, E.J., Schothorst, R.C., van Egmond, H.P., 2002. Toxicology and Occurrence of Nivalenol, Fusarenon X, Diacetoxyscirpenol, Neosolaniol and 3- and 15-acetyldeoxynivalenol: a Review of Six Trichothecenes. Dutch National Institute for Public Health and the Environment Repository, Report Number 388802024 . http://rivm.openrepository.com/rivm/handle/10029/9184.

Šegvić Klarić, M., 2012. Adverse Effects Of Combined Mycotoxins/Štetni Učinci Kombiniranih Mikotoksina. Archives of Industrial Hygiene and Toxicology 63, 519-530. 
Sintov, A., Bialer, M., Yagen, B., 1986. Pharmacokinetics of T-2 toxin and its metabolite HT-2 toxin, after intravenous administration in dogs. Drug metabolism and disposition 14, 250-254.

Sobrova, P., Adam, V., Vasatkova, A., Beklova, M., Zeman, L., Kizek, R., 2010. Deoxynivalenol and its toxicity. Interdisciplinary toxicology 3, 94.

Stadler, M., Bardiau, F., Seidel, L., Albert, A., Boogaerts, J.G., 2003. Difference in risk factors for postoperative nausea and vomiting. The Journal of the American Society of Anesthesiologists 98, 46-52.

Stanciu, O., Banc, R., Cozma, A., Filip, L., Miere, D., Mañes, J., Loghin, F., 2015. Occurence of Fusarium Mycotoxins in Wheat from Europe-A Review. Acta Universitatis Cibiniensis. Series E: Food Technology $19,35-60$.

Steinberg, E.B., Henderson, A., Karpati, A., Hoekstra, M., Marano, N., Souza, J.M., Simons, M., Kruger, K., Giroux, J., Rogers, H.S., 2006. Mysterious outbreaks of gastrointestinal illness associated with burritos supplied through school lunch programs. Journal of Food Protection 69, 1690-1698.

Van den Berg, M., Birnbaum, L., Bosveld, A., Brunström, B., Cook, P., Feeley, M., Giesy, J.P., Hanberg, A., Hasegawa, R., Kennedy, S.W., 1998. Toxic equivalency factors (TEFs) for PCBs, PCDDs, PCDFs for humans and wildlife. Environmental Health Perspectives 106, 775-792.

Van den Berg, M., Birnbaum, L.S., Denison, M., De Vito, M., Farland, W., Feeley, M., Fiedler, H., Hakansson, H., Hanberg, A., Haws, L., 2006. The 2005 World Health Organization reevaluation of human and mammalian toxic equivalency factors for dioxins and dioxin-like compounds. Toxicological Sciences $93,223-241$.

Veršilovskis, A., Geys, J., Huybrechts, B., Goossens, E., De Saeger, S., Callebaut, A., 2012. Simultaneous determination of masked forms of deoxynivalenol and zearalenone after oral dosing in rats by LCMS/MS. World Mycotoxin Journal 5, 303-318.

Visconti, A., Minervini, F., Lucivero, G., Gambatesa, V., 1991. Cytotoxic and immunotoxic effects of Fusarium mycotoxins using a rapid colorimetric bioassay. Mycopathologia 113, 181-186. 
Wu, Q., Dohnal, V., Kuca, K., Yuan, Z., 2013. Trichothecenes: structure-toxic activity relationships. Current Drug Metabolism 14, 641-660.

Wu, W., Bates, M., Bursian, S.J., Link, J.E., Flannery, B.M., Sugita-Konishi, Y., Wantanabe, M., Zhang, H., Pestka, J.J., 2012a. Comparison of emetic potencies of the 8-ketotrichothecenes deoxynivalenol, 15acetyldeoxynivalenol, 3-acetyldeoxynivalenol, fusarenon $\mathrm{X}$ and nivalenol. Toxicological Sciences 131, 279-291.

Wu, W., Bates, M.A., Bursian, S.J., Flannery, B., Zhou, H.R., Link, J.E., Zhang, H., Pestka, J.J., 2013. Peptide YY3-36 and 5-Hydroxytryptamine Mediate Emesis Induction by the Trichothecene Deoxynivalenol (Vomitoxin). Toxicological Sciences 133, 186-195.

Wu, W., Flannery, B.M., Sugita-Konishi, Y., Watanabe, M., Zhang, H., Pestka, J.J., 2012b. Comparison of murine anorectic responses to the 8-ketotrichothecenes 3-acetyldeoxynivalenol, 15acetyldeoxynivalenol, fusarenon $X$ and nivalenol. Food and Chemical Toxicology 50, 2056-2061.

Wu, W., Zhou, H.-R., Bursian, S.J., Pan, X., Link, J.E., Berthiller, F., Adam, G., Krantis, A., Durst, T., Pestka, J.J., 2014. Comparison of Anorectic and Emetic Potencies of Deoxynivalenol (Vomitoxin) to the Plant Metabolite Deoxynivalenol-3-Glucoside and Synthetic Deoxynivalenol Derivatives EN139528 and EN139544. Toxicological Sciences 142, 167-181.

Yoshizawa, T., 1983. Trichothecenes: chemical, biological, and toxicological aspects. Kodansha Ltd.: Tokyo, Japan, pp. 195-209.

Zhou, T., He, J., Gong, J., 2008. Microbial transformation of trichothecene mycotoxins. World Mycotoxin Journal 1, 23-30. 


\section{TABLES}

Table 1. IP injection: Summary of results for the emesis endpoint using a mink animal model. Different doses of trichothecenes were administered to the animals by IP injection: $\mathrm{N}=4$ for Type $\mathrm{A}$ and $\mathrm{N}=6$ for Type B trichothecenes. The data presented were generated by studies conducted by Wu et al. (2012a) and reported in an earlier publication. HT-2 (HT-2 toxin), T-2 (T-2 toxin), DON (deoxynivalenol), 3-ADON (3-acetyldeoxynivalenol), 15-ADON (15-acetyldeoxynivalenol), NIV (nivalenol), FX (fusarenon-X).

\begin{tabular}{|c|c|c|c|c|c|c|c|}
\hline \multirow[b]{2}{*}{ Toxin } & \multirow{2}{*}{$\begin{array}{c}\text { Doses } \\
(\mathrm{mg} / \mathrm{kg} \mathrm{bw})\end{array}$} & \multirow[b]{2}{*}{ Incidence } & \multirow{2}{*}{$\begin{array}{l}\text { Latency } \\
(\min )\end{array}$} & \multirow{2}{*}{$\begin{array}{l}\text { Emesis duration } \\
\qquad(\min )\end{array}$} & \multicolumn{2}{|c|}{ Number of emetic events } & \multirow[b]{2}{*}{ Total } \\
\hline & & & & & Retching & Vomiting & \\
\hline \multirow[t]{5}{*}{ HT-2 } & 0 & $0 / 4$ & & & & & \\
\hline & 0.001 & $0 / 4$ & & & & & \\
\hline & 0.01 & $0 / 4$ & & & & & \\
\hline & 0.05 & $1 / 4$ & $27.5 \pm 0$ & $70.5 \pm 0$ & $19.5 \pm 19.5$ & $2.8 \pm 2.8$ & $22.3 \pm 22.3$ \\
\hline & 0.25 & $4 / 4$ & $23.8 \pm 1.6$ & $242.1 \pm 6.6$ & $210 \pm 21.5$ & $27.8 \pm 2$ & $237.8 \pm 22.2$ \\
\hline \multirow[t]{5}{*}{$\mathrm{T}-2$} & 0 & $0 / 4$ & & & & & \\
\hline & 0.001 & $0 / 4$ & & & & & \\
\hline & 0.01 & $0 / 4$ & & & & & \\
\hline & 0.05 & $1 / 4$ & $30 \pm 0$ & $55 \pm 0$ & $17.5 \pm 17.5$ & $2.3 \pm 2.3$ & $19.8 \pm 19.8$ \\
\hline & 0.25 & $4 / 4$ & $27 \pm 3.2$ & $206.5 \pm 7.2$ & $161.3 \pm 15.1$ & $22.5 \pm 2.3$ & $183.8 \pm 17.2$ \\
\hline \multicolumn{8}{|l|}{$3-$} \\
\hline \multicolumn{8}{|l|}{$\mathrm{ADO}$} \\
\hline \multirow[t]{5}{*}{$\mathrm{N}$} & 0 & $0 / 3$ & - & - & $0 \pm 0$ & $0 \pm 0$ & $0 \pm 0$ \\
\hline & 0.1 & $0 / 6$ & - & - & $0 \pm 0$ & $0 \pm 0$ & $0 \pm 0$ \\
\hline & 0.2 & $3 / 6$ & $36 \pm 14$ & $3 \pm 2$ & $7 \pm 4$ & $2 \pm 1$ & $9 \pm 5$ \\
\hline & 0.3 & $5 / 6$ & $48 \pm 9$ & $26 \pm 7$ & $28 \pm 8$ & $6 \pm 2$ & $34 \pm 9$ \\
\hline & 0.4 & $6 / 6$ & $19 \pm 4$ & $54 \pm 2$ & $81 \pm 10$ & $16 \pm 1$ & $97 \pm 11$ \\
\hline \multicolumn{8}{|l|}{$15-$} \\
\hline $\mathrm{ADO}$ & 0 & $0 / 5$ & - & - & $0 \pm 0$ & $0 \pm 0$ & $0 \pm 0$ \\
\hline
\end{tabular}




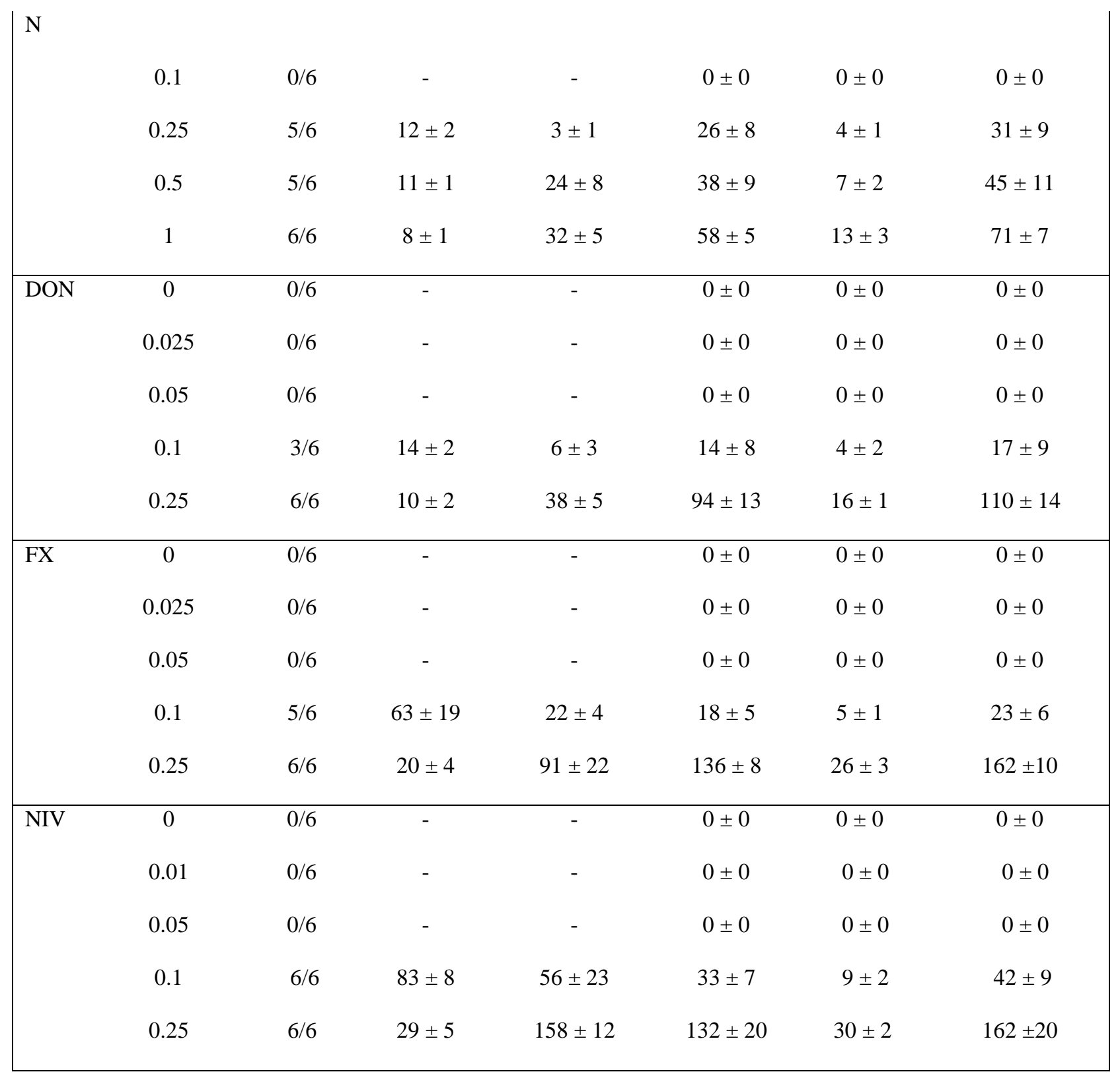


Table 2. Gavage: Summary of results for the emesis end-point. Various doses of trichothecenes were administered to the mink animal model using the gavage method. $\mathrm{N}=4$ for Type $\mathrm{A}$ and $\mathrm{N}=6$ for Type $\mathrm{B}$ trichothecenes. The data presented were generated by studies conducted by Wu et al. (2012a) and reported in an earlier publication. HT-2 (HT-2 toxin), T-2 (T-2 toxin), DON (deoxynivalenol), 3-ADON (3-acetyldeoxynivalenol), 15-ADON (15-acetyldeoxynivalenol), NIV (nivalenol), FX (Fusarenon X).

\begin{tabular}{|c|c|c|c|c|c|c|c|}
\hline \multirow[b]{2}{*}{ Toxin } & \multirow{2}{*}{$\begin{array}{c}\text { Doses } \\
\text { (mg/kg bw) }\end{array}$} & \multirow[b]{2}{*}{ Incidence } & \multirow{2}{*}{$\begin{array}{l}\text { Latency } \\
\text { (min) }\end{array}$} & \multirow{2}{*}{$\begin{array}{l}\text { Emesis } \\
\text { duration } \\
\text { (min) }\end{array}$} & \multicolumn{3}{|c|}{ Number of emetic events } \\
\hline & & & & & Retching & Vomiting & Total \\
\hline \multirow[t]{5}{*}{ HT-2 } & 0 & $0 / 4$ & & & & & \\
\hline & 0.005 & $0 / 4$ & & & & & \\
\hline & 0.05 & $3 / 4$ & $27 \pm 7.1$ & $49.7 \pm 11.2$ & $60.3 \pm 24.4$ & $7.3 \pm 2.9$ & $67.5 \pm 27.3$ \\
\hline & 0.25 & $4 / 4$ & $21.6 \pm 3.8$ & $120.6 \pm 21.2$ & $157.3 \pm 24.2$ & $17.8 \pm 2.4$ & $175 \pm 26.4$ \\
\hline & 0.5 & $4 / 4$ & $19.1 \pm 1.8$ & $200.6 \pm 18.1$ & $222.3 \pm 28.5$ & $23 \pm 3.2$ & $245.3 \pm 31.2$ \\
\hline \multirow[t]{5}{*}{$\mathrm{T}-2$} & 0 & $0 / 4$ & & & & & \\
\hline & 0.005 & $0 / 4$ & & & & & \\
\hline & 0.05 & $3 / 4$ & $22.5 \pm 2.6$ & $52.3 \pm 15.4$ & $66.3 \pm 22.6$ & $8 \pm 3.1$ & $74.3 \pm 25.5$ \\
\hline & 0.25 & $4 / 4$ & $19.5 \pm 3.3$ & $108 \pm 11.1$ & $130 \pm 16.9$ & $17.3 \pm 1.3$ & $147.3 \pm 18.1$ \\
\hline & 0.5 & $4 / 4$ & $13.1 \pm 3.2$ & $170.9 \pm 22.1$ & $191.3 \pm 50.4$ & $21 \pm 2.6$ & $212.3 \pm 52.7$ \\
\hline \multirow[t]{5}{*}{ 3-ADON } & 0 & $0 / 6$ & - & - & $0 \pm 0$ & $0 \pm 0$ & $0 \pm 0$ \\
\hline & 0.05 & $0 / 6$ & - & - & $0 \pm 0$ & $0 \pm 0$ & $0 \pm 0$ \\
\hline & 0.25 & $1 / 6$ & $62 \pm 0$ & $1 \pm 0$ & $2 \pm 2$ & $1 \pm 1$ & $3 \pm 3$ \\
\hline & 0.5 & $5 / 6$ & $53 \pm 9$ & $12 \pm 9$ & $16 \pm 8$ & $4 \pm 1$ & $20 \pm 9$ \\
\hline & 1 & $6 / 6$ & $19 \pm 4$ & $44 \pm 9$ & $65 \pm 11$ & $14 \pm 2$ & $79 \pm 13$ \\
\hline \multirow[t]{2}{*}{ 15-ADON } & 0 & $0 / 6$ & - & - & $0 \pm 0$ & $0 \pm 0$ & $0 \pm 0$ \\
\hline & 0.01 & $0 / 6$ & - & - & $0 \pm 0$ & $0 \pm 0$ & $0 \pm 0$ \\
\hline
\end{tabular}




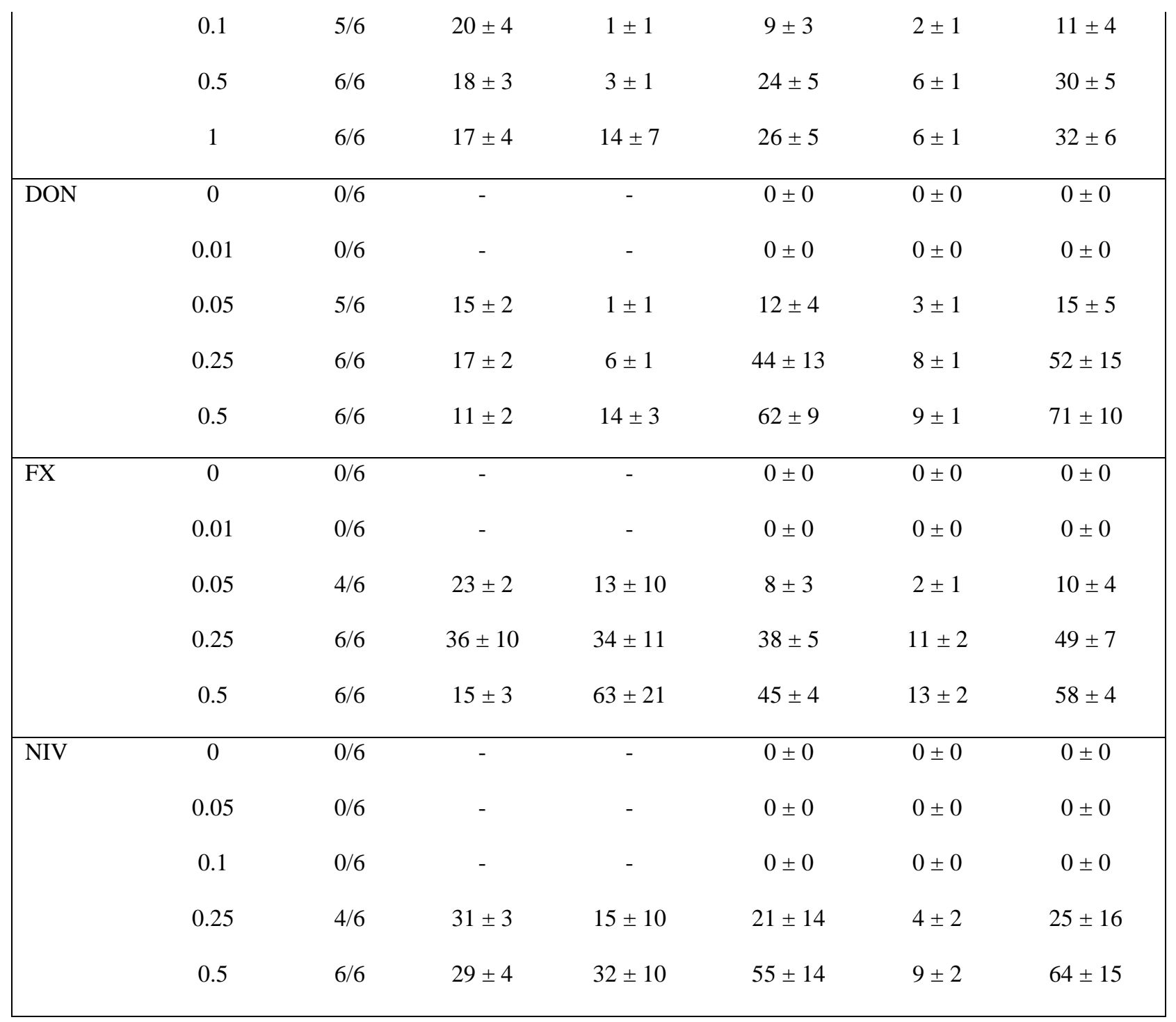


Table 3. The emetic benchmark doses and relative potencies of type A and type B trichothecenes administered to mink using gavage (oral) and IP dosing methods. Relative emetic potencies are the ratio of the BMD of DON (the most prevalent and widely studied trichothecene) to the BMD of each of the other trichothecenes studied. DON (deoxynivalenol), 3-ADON (3-acetyldeoxynivalenol), 15-ADON (15acetyldeoxynivalenol), NIV (nivalenol), FX (Fusarenon X), HT-2 (HT-2 toxin), T-2 (T-2 toxin).

\begin{tabular}{|c|c|cc|cc|}
\hline & & IP BMD & Relative & Gavage BMD & Relative \\
Type & Trichothecene & $(\mu \mathrm{g} / \mathbf{k g}$ bw $)$ & potency & $(\boldsymbol{\mu g} / \mathbf{k g ~ b w})$ & potency \\
\hline A & HT-2 & 31 & 2.38 & 14 & 1.73 \\
A & T-2 & 31 & 2.38 & 14 & 1.73 \\
B & $3-A D O N$ & 141 & 0.52 & 198 & 0.12 \\
B & 15-ADON & 108 & 0.67 & 40 & 0.60 \\
B & DON & 73 & 1.00 & 24 & 1.00 \\
B & FX & 60 & 1.22 & 23 & 1.04 \\
B & NIV & 63 & 1.16 & 141 & 0.17 \\
\hline
\end{tabular}


Table 4. The number of emetic responses by mink administered a fixed oral dose $(0.5 \mathrm{mg} / \mathrm{kg}$ bw $)$ of type A and type B trichothecenes. The $0.5 \mathrm{mg} / \mathrm{kg}$ oral dietary dose was the common dose included in all animal sub-studies conducted by Wu et al. (2012a) when investigating the emetic responses of mink to a range of doses of trichothecenes. The frequency of retching and vomiting were counted as discrete events. The total number of emetic events was calculated as the sum of retching and vomiting. Total emetic events recorded as Means \pm SD. Different letters superscripted under "Total" indicates those groups are significantly different $(\mathrm{p} \leq 0.05)$.

\begin{tabular}{|c|c|c|c|c|}
\hline \multirow{2}{*}{ Type } & \multirow{2}{*}{ Trichothecene } & \multicolumn{3}{|c|}{ Number of emetic events } \\
\cline { 2 - 4 } & Retching & Vomiting & Total \\
\hline A & HT-2 & 222 & 23 & $245 \pm 32^{\mathrm{a}}$ \\
A & T-2 & 191 & 21 & $212 \pm 53^{\mathrm{a}}$ \\
B & $3-$ ADON & 16 & 4 & $20 \pm 9^{\mathrm{c}}$ \\
B & $15-$ ADON & 24 & 6 & $30 \pm 5^{\mathrm{c}}$ \\
B & DON & 62 & 9 & $71 \pm 10^{\mathrm{b}}$ \\
B & FX & 45 & 13 & $58 \pm 4^{\mathrm{b}}$ \\
B & NIV & 55 & 9 & $64 \pm 15^{\mathrm{b}}$ \\
\hline
\end{tabular}

DON (deoxynivalenol), 3-ADON (3-acetyldeoxynivalenol), 15-ADON (15-acetyldeoxynivalenol), NIV (nivalenol), FX (Fusarenon X), HT-2 (HT-2 toxin), T-2 (T-2 toxin). 


\section{FIGURES}

Figure 1. The general structure of trichothecenes and the various functional groups

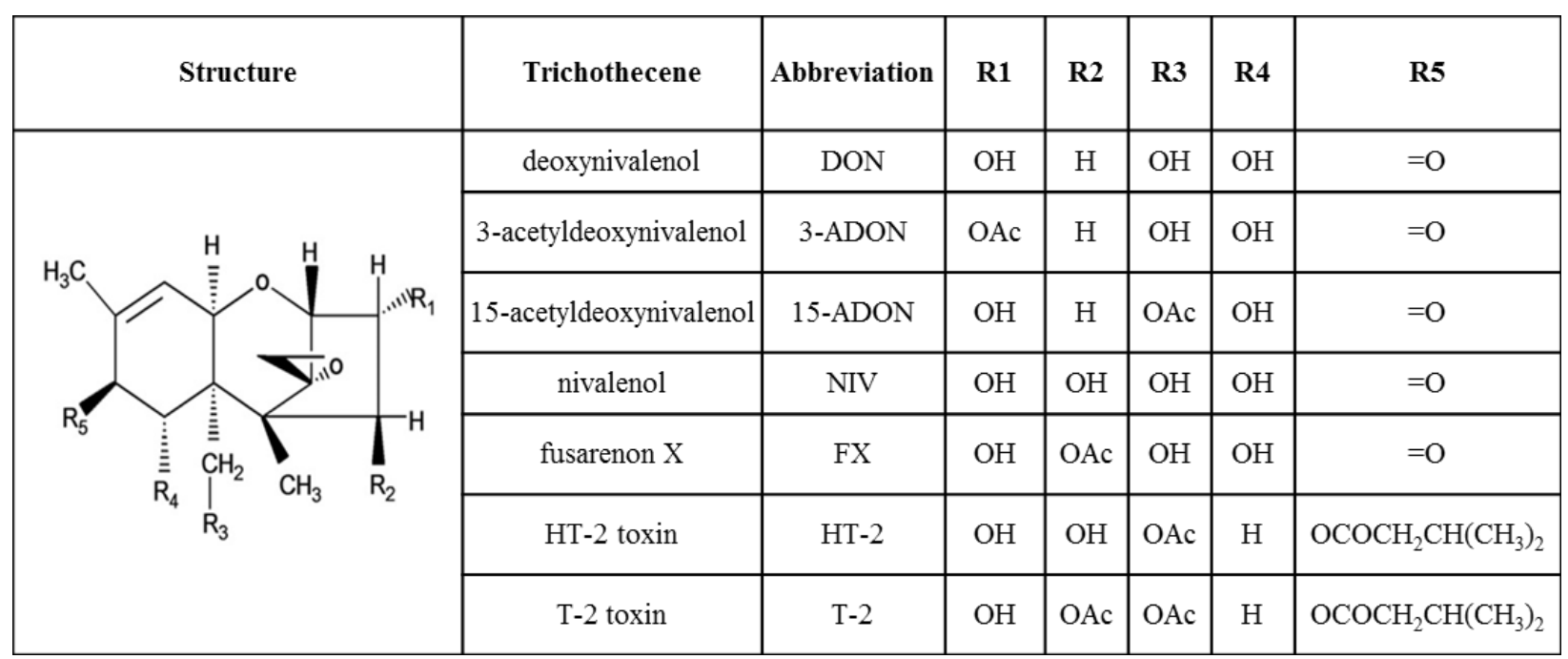

Figure 2. Experimental design for IP or oral gavage dosing mink emesis study, adapted from Wu et al. (2012).

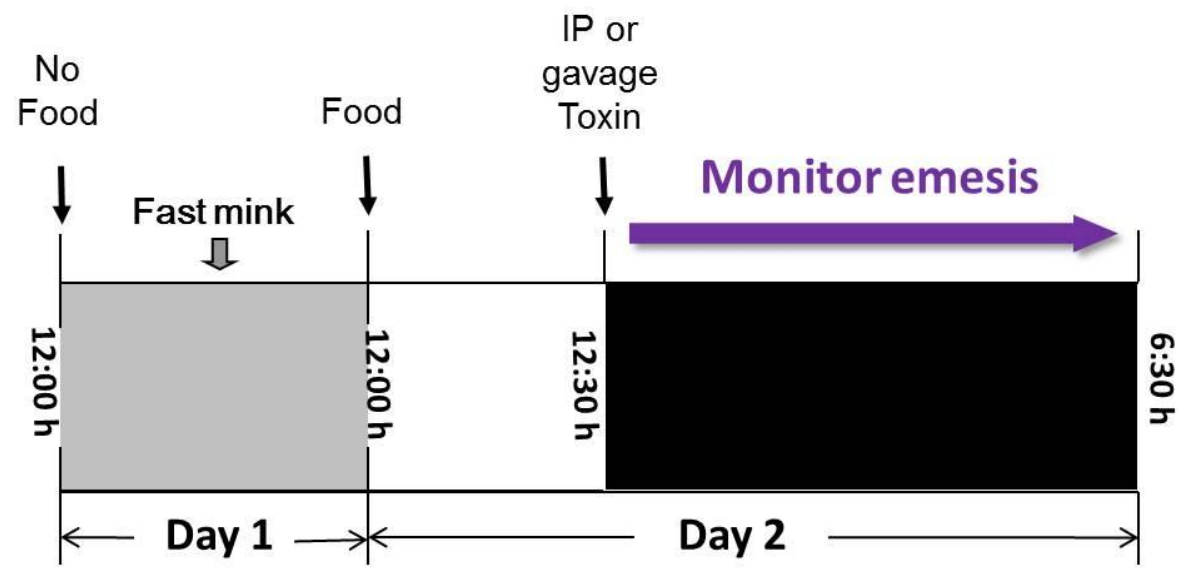

